\title{
RESEARCH
}

Open Access

\section{Aortic flow patterns and wall shear stress maps by 4D-flow cardiovascular magnetic resonance in the assessment of aortic dilatation in bicuspid aortic valve disease}

José Fernando Rodríguez-Palomares ${ }^{1 *+}$, Lydia Dux-Santoy ${ }^{1 \dagger}$, Andrea Guala, Raquel Kale ${ }^{1}$, Giuliana Maldonado ${ }^{1}$, Gisela Teixidó-Turà', Laura Galian', Marina Huguet², Filipa Valente', Laura Gutiérrez', Teresa González-Alujas', Kevin M. Johnson ${ }^{3}$, Oliver Wieben ${ }^{3}$, David García-Dorado ${ }^{1}$ and Arturo Evangelista ${ }^{1}$

\begin{abstract}
Background: In patients with bicuspid valve (BAV), ascending aorta (AAo) dilatation may be caused by altered flow patterns and wall shear stress (WSS). These differences may explain different aortic dilatation morphotypes. Using 4Dflow cardiovascular magnetic resonance (CMR), we aimed to analyze differences in flow patterns and regional axial and circumferential WSS maps between BAV phenotypes and their correlation with ascending aorta dilatation morphotype.

Methods: One hundred and one BAV patients (aortic diameter $\leq 45 \mathrm{~mm}$, no severe valvular disease) and 20 healthy subjects were studied by 4D-flow CMR. Peak velocity, flow jet angle, flow displacement, in-plane rotational flow (IRF) and systolic flow reversal ratio (SFRR) were assessed at different levels of the AAo. Peak-systolic axial and circumferential regional WSS maps were also estimated. Unadjusted and multivariable adjusted linear regression analyses were used to identify independent correlates of aortic root or ascending dilatation. Age, sex, valve morphotype, body surface area, flow derived variables and WSS components were included in the multivariable models.
\end{abstract}

Results: The AAo was non-dilated in 24 BAV patients and dilated in 77 (root morphotype in 11 and ascending in 66). BAV phenotype was right-left (RL-) in 78 patients and right-non-coronary (RN-) in 23. Both BAV phenotypes presented different outflow jet direction and velocity profiles that matched the location of maximum systolic axial WSS. RL-BAV velocity profiles and maximum axial WSS were homogeneously distributed right-anteriorly, however, RN-BAV showed higher variable profiles with a main proximal-posterior distribution shifting anteriorly at mid-distal AAo. Compared to controls, BAV patients presented similar WSS magnitude at proximal, mid and distal AAo $(p=0.764,0.516$ and 0.053 , respectively) but lower axial and higher circumferential WSS components ( $p<0.001$ for both, at all aortic levels). Among BAV patients, RN-BAV presented higher IRF at all levels ( $p=0.024$ proximal, 0.046 mid and 0.002 distal AAo) and higher circumferential WSS at mid and distal AAo ( $p=0.038$ and 0.046 , respectively) than RL-BAV. However, axial WSS was higher in RL-BAV compared to RN-BAV at proximal and mid AAo $(p=0.046,0.019$, respectively). Displacement and axial WSS were independently associated with the root-morphotype, and circumferential WSS and SFRR with the ascending-morphotype.

(Continued on next page)

\footnotetext{
*Correspondence: jfrodriguezpalomares@gmail.com; jfrodrig@vhebron.net

'Equal contributors

${ }^{1}$ Hospital Universitari Vall d'Hebron, Department of Cardiology. Vall d'Hebron

Institut de Recerca (VHIR), Universitat Autònoma de Barcelona, Paseo Vall

d'Hebron 119-129, 08035 Barcelona, Spain

Full list of author information is available at the end of the article
}

(c) The Author(s). 2018 Open Access This article is distributed under the terms of the Creative Commons Attribution 4.0 International License (http://creativecommons.org/licenses/by/4.0/), which permits unrestricted use, distribution, and reproduction in any medium, provided you give appropriate credit to the original author(s) and the source, provide a link to the Creative Commons license, and indicate if changes were made. The Creative Commons Public Domain Dedication waiver (http://creativecommons.org/publicdomain/zero/1.0/) applies to the data made available in this article, unless otherwise stated. 
(Continued from previous page)

Conclusions: Different BAV-phenotypes present different flow patterns with an anterior distribution in RL-BAV, whereas, RN-BAV patients present a predominant posterior outflow jet at the sinotubular junction that shifts to anterior or right anterior in mid and distal AAo. Thus, RL-BAV patients present a higher axial WSS at the aortic root while RN-BAV present a higher circumferential WSS in mid and distal AAo. These results may explain different AAo dilatation morphotypes in the BAV population.

Keywords: Bicuspid aortic valve, 4D flow cardiovascular magnetic resonance (4D flow CMR), Wall shear stress, Ascending aorta, Aorta hemodynamics, Aortic dilatation

\section{Background}

Bicuspid aortic valve (BAV) is the most common congenital valvular abnormality, occurring in $1-2 \%$ of the general population [1]. Between 60 and $80 \%$ BAV patients develop aortic dilatation that is associated with an increased risk of aortic dissection and rupture $[2,3]$. Aortic diameter alone has proved to be largely ineffective to predict these complications [4-6].

The most common BAV fusion phenotype involves the right and left cusps (RL-BAV) and is associated with dilatation of the tubular ascending aorta (AAo) and aortic root primarily along the convexity of the aorta. While the fusion of the right and non-coronary cusps (RN-BAV) induces arch dilatation with involvement of the tubular ascending aorta, with relative sparing of the root [3]. However, not all patients with the same BAV phenotype have the same pattern of aortopathy and, furthermore, $26-35 \%$ of BAV present a non-dilated aorta [2]. Therefore, other factors beyond valve phenotype may be related to aortic dilatation. Although controversy exists regarding the influence of hemodynamic $[7,8]$ and genetic factors in aortic dilatation [9], different studies have provided significant evidence that altered outflow pattern is related to aortic morphology $[7,10]$.

In recent years, time-resolved three-dimensional phasecontrast cardiovascular magnetic resonance (CMR 4D-flow) has emerged as a potential tool to provide comprehensive information on aortic hemodynamics with 3D visualization of blood-flow patterns [11, 12]. Using 4D-flow, several studies have analyzed flow and wall shear stress (WSS) on specific analysis planes in the ascending aorta $[7,11,13$, 14], and their relation to aortic dilatation [7]. Although some studies have analyzed WSS components [11, 13], their eventual association to different dilatation morphotype has not been investigated [11]. Only one study analyzed a BAV population differentiating between dilatation morphotype, but the computed WSS was not referenced to local direction [7]. Additionally, it has recently been shown that regions with increased WSS correspond to extracellular matrix dysregulation and elastic fiber degeneration in the ascending aorta and contribute to the development of aortopathy $[8,15,16]$. Thus, a more detailed 3D representation of WSS components $[12,17]$ may help to explain the different aortic dilatation morphotypes. However, there is not yet sufficient evidence to include these variables for clinical management $[7,11]$.

The aim of our study was to assess the relation between aortic flow patterns and axial and circumferential WSS by $4 \mathrm{D}$ flow CMR through the entire ascending aorta in a large BAV population, and to establish their association with aortic dilatation and morphotype.

\section{Methods \\ Study population}

Patients with RL- or RN-BAV phenotype, aortic root and AAo diameters $\leq 45 \mathrm{~mm}$ and no severe valvular disease (aortic regurgitation $\leq$ Grade III; aortic velocity $<3 \mathrm{~m} / \mathrm{s}$ ) by echo were consecutively and prospectively recruited. Inclusion criteria were: age $>18$ years, no cardiovascular disease, sinus rhythm, no hypertension, no connective tissue disorders, no aortic coarctation or other congenital heart diseases and no contraindication for CMR. Also, 20 healthy subjects matched with the BAV population in age and aortic diameters were studied. The study was approved by the local ethics committee and informed consent was obtained from all participants.

\section{Cardiovascular magnetic resonance protocol}

CMR studies were performed on a $1.5 \mathrm{~T}$ scanner (Signa, General Electric Healthcare, Waukesha, Wisconsin, USA). The protocol included 2D balanced steady-state freeprecession (bSSFP) cine imaging which was used to assess BAV phenotype and aortic diameters (using the doubleoblique multiplanar reconstruction), and a 4D-flow acquisition with retrospective electrocardiogram (ECG)-gating during free-breathing. Endovenous contrast was not given.

For 4D-flow CMR, phase-contrast (PC) VIPR sequence [18], a radially undersampled acquisition with 5-point balanced velocity encoding was used [19]. The acquisition volume was set to include the entire thoracic aorta. Acquisitions were made with an eight-channel cardiac coil (HD Cardiac, GE Healthcare) using the following parameters: velocity encoding (VENC) $200 \mathrm{~cm} / \mathrm{s}$, field of view (FOV) 400x400x400 mm, scan matrix $160 \times 160 \times$ 160 (voxel size of $2.5 \times 2.5 \times 2.5 \mathrm{~mm}^{3}$ ), flip angle $8^{\circ}$, repetition time $4.2-6.4 \mathrm{~ms}$ and echo time $1.9-3.7 \mathrm{~ms}$. 
This data set was reconstructed the nominal temporal resolution of each patient and was (5xTR) $21 \mathrm{~ms}-32 \mathrm{~ms}$. Reconstructions were performed offline with corrections for background phase from concomitant gradients and eddy currents, and trajectory errors of the 3D radial acquired k-space [19, 20].

\section{Data analysis: 4D flow data processing}

Eight double-oblique analysis planes were equally distributed in the AAo between the sinotubular junction and the origin of the brachiocephalic trunk (see red and blue lines on the left side of Fig. 1b). The vessel lumen was manually segmented in every analysis plane for all systolic phases using an angiogram derived from the 4D-flow data using complex difference processing [21]. Mass Research Software (Leiden University Medical Center, Leiden, the Netherlands) was used for the location of the analysis planes and lumen segmentation. Lumen contour points and 3D velocity data for each plane were exported for calculations to be made using custom Matlab software (MathWorks Inc., Natick, Massachusetts, USA).

\section{Flow parameters}

Peak velocity, flow jet angle, normalized flow displacement, and in-plane rotational flow were calculated at 3 different levels at the proximal, mid and distal AAo (blue lines on Fig. 1b). Flow parameters were averaged using 1 time-frame before and 2 frames after peak systole to mitigate noise.

Flow jet angle and normalized flow displacement were obtained as described by Sigovan et al. [22]. In-plane rotational flow was quantified as the through-plane component $\left(\Gamma_{\mathrm{T}}\right)$ of circulation $(\Gamma)$, which is a parameter used in fluid dynamics to quantify rotation of flow within a plane. To this aim, vorticity $(\omega)$ was computed in each double-oblique analysis plane and circulation $(\Gamma)$ was obtained as the integral of vorticity with respect to cross-sectional area, $\Gamma=\iint \omega d S[23]$.

Flow volumes were calculated as the time-integral over systolic phases of forward and backward through-plane flow rate curves, and used for the calculation of systolic flow reversal ratio (SFRR) [24] at mid and distal AAo (Figs. 1 and 2).

$$
\operatorname{SFRR}(\%)=\frac{\int_{0}^{T_{s}} v_{S B F}(t) d t}{\int_{0}^{T_{s}} v_{S F F}(t) d t} \cdot 100
$$

Where $v_{S F F}$ and $v_{S B F}$ represent the forward and backward flow rates, respectively, and $T_{s}$ is systolic time interval.

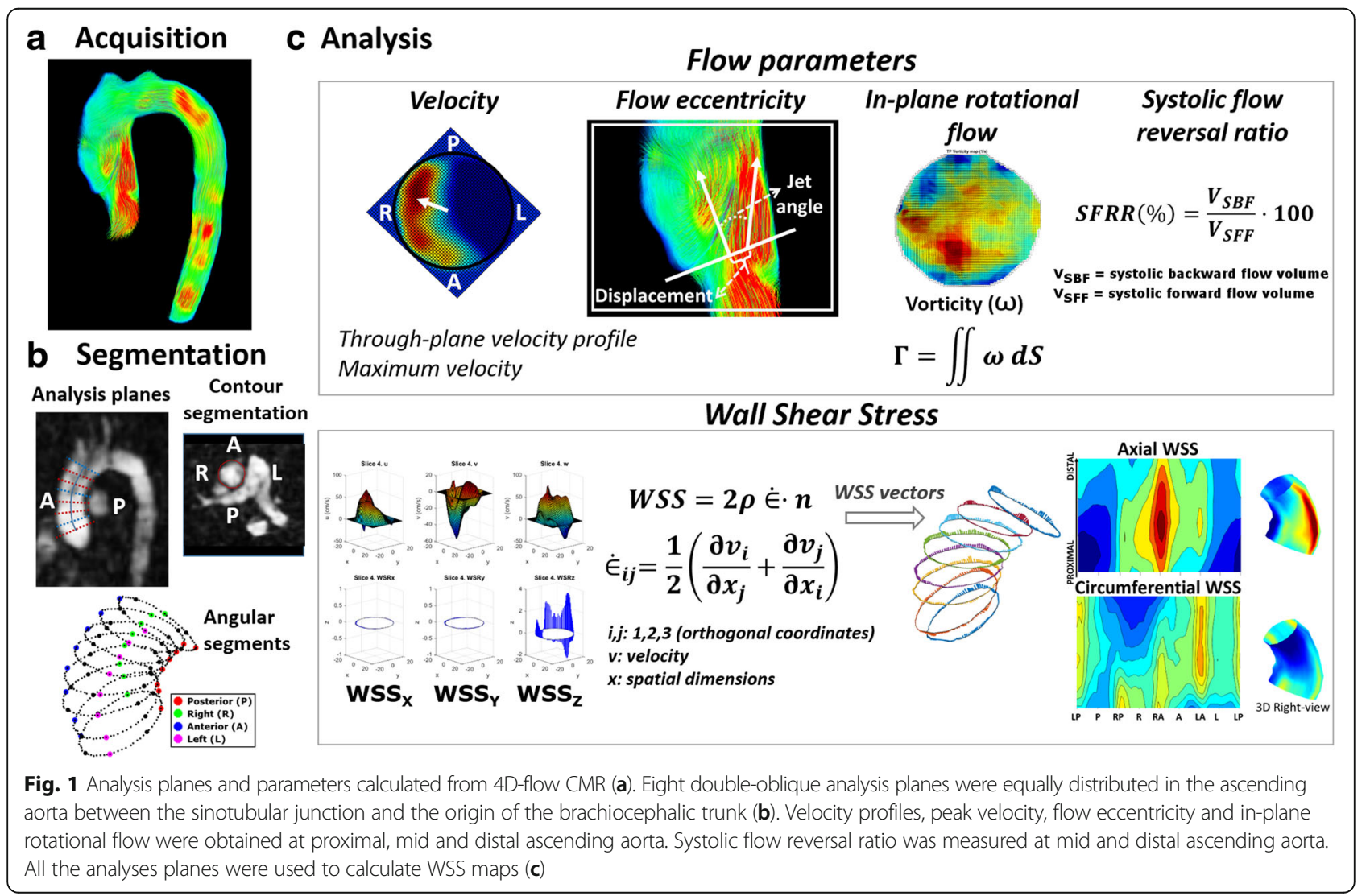




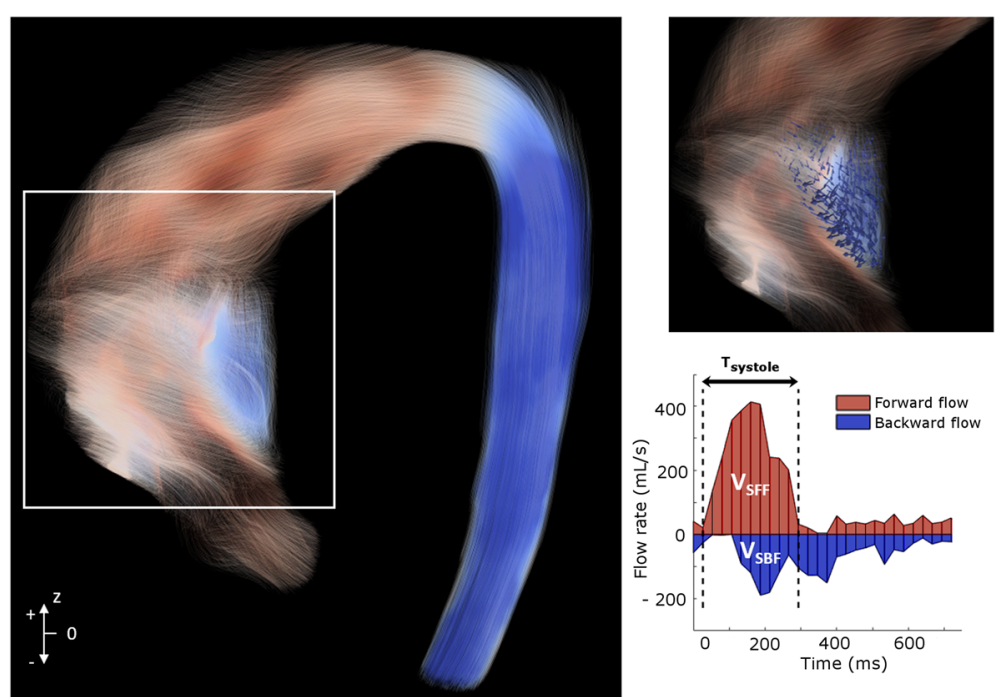

Fig. 2 Systolic backward flow in a BAV patient. Red streamlines indicate forward flow in the ascending aorta, while blue streamlines indicate systolic backward flow. SFRR: systolic flow reversal ratio, $V_{\text {SFF: }}$ total systolic forward flow, $V_{\text {SBF }}$ : total systolic backward flow

\section{Wall shear stress}

Peak-systolic WSS vectors (averaged from 1-time frame before, and 2-time frames after peak) were calculated at 64 points equally distributed along the aortic lumen for the 8 cross sectional analysis planes by fitting the 3D velocity data with $\mathrm{B}$-spline surfaces and computing velocity derivatives on the segmented vessel lumen [25]. WSS vectors were decomposed in their through-plane (axial) and in-plane (circumferential) components.

Contour-averaged magnitude (WSS mag,avg $_{\text {) and WS }}$ components WSS $_{\mathrm{ax}, \text { avg }}$ and $\mathrm{WSS}_{\text {circ,avg }}$ ) were calculated at proximal, mid and distal AAo.

Averaged WSS maps were obtained for each BAV phenotype and dilatation morphotype (Figs. 1c, 6 and 8). To this aim, the 64 points of the lumen contour per cross-sectional plane were aligned for all patients using the inner aortic curvature as a reference. Averaged WSS maps were calculated by computing point-to-point WSS means for all the 8 sections analyzed. Finally, statistical significance maps of axial and circumferential WSS were calculated for the mean WSS value for 8 standardized angular segments [14] of the aortic wall. Averaged WSS maps and statistical significance maps were visualized using bilinear interpolation.

\section{Dilatation morphotypes}

In order to determine the presence of aortic root or ascending dilatation, aortic diameters were adjusted with a logarithm transformation to set the z-score for both sinuses (zsinus) and ascending aorta (zAscAo) accounting for sex, age and body surface area (BSA) as described by Campens et al. [26]. Using a z-score cutoff for aortic dilatation of 2 standard error of estimate, patients were categorized according to the tract predominantly or exclusively involved in dilatation according to Della Corte's classification [2]. Thus, patients were classified as nondilated (zsinus $\leq 2$ and $z A s c A o \leq 2$ ), root-morphotype (zsinus $>2$ and zsinus $>$ zAscAo) or ascending-morphotype (zAscAo $>2$ and zAscAo $>$ zsinus).

\section{Statistical analysis}

Continuous demographic variables were expressed as mean \pm standard deviation. The Kolmogorov-Smirnov test was used to evaluate the normality distribution of variables. Differences between groups for continuous parameters were assessed by Student's t-test if they presented a normal distribution or ANOVA with Bonferroni correction for multiple comparisons, and Mann-Whitney U test if they did not present a normal distribution. For categorical variables, general characteristics of the sample were assessed by percentages (chi-square test). Logarithmic transformation (ln) was performed for variables with both positive and negative values (such as circulation and WSS) to preserve the distinction between negative, zero and positive values as described by Whittaker et al. [27].

Multivariable logistic regression analyses with a forward selection procedure were used to evaluate specific relations between demographic and flow variables and aortic root or ascending dilatation. Variables were entered into the model if $P<0.25$ in univariate analyses. The aortic root morphotype was compared to the rest of groups (non-dilated and ascending), whereas, the ascending group was compared to non-dilated and root morphotype as described elsewhere [28]. To avoid multicollinearity, variables were excluded from the multivariable logistic regression if the tolerance test was $<0.1$ or the variation inflation factor $>5$. This is 
the case of the same flow variables computed at different locations. The variables entered the multivariable model were chosen as those demonstrating better predictive value, i.e. those having higher AUC in the Receiver operating characteristic (ROC) curve, as compared to the same variable computed at another location. ROC curves were performed to assess the relationship between variables obtained in the multivariable analysis and aortic morphotypes.

A two-tailed $P$ value $<0.05$ was considered statistically significant. SPSS (version 19.0, International Business Machines, Armonk, New York, USA) was used for the analysis.

\section{Results}

One hundred and one BAV patients (78 RL- and $23 \mathrm{RN}$ phenotype) and 20 healthy subjects completed the study protocol. Demographic characteristics and aortic diameters among groups are shown in Table 1. Demographics did not significantly differ among the three groups (controls, RN-BAV, RL-BAV). Although aortic diameters were larger in BAV compared to controls, no statisticallysignificant differences were observed. However, z-scores were higher in the RL-BAV phenotype at the sinuses of Valsalva and at the AAo in the RN-BAV.

In the RL-BAV group $(n=78), 20$ patients $(25.6 \%)$ presented a non-dilated, $10(12.8 \%)$ a root- and 48 (61.5\%) an ascending-morphotype aorta. In the RN-BAV group $(n=23), 4$ patients $(17.4 \%)$ presented a non-dilated, $1(4.3 \%)$ a root- and $18(78.3 \%)$ an ascending-morphotype. Only three of the patients with root-morphotype did not present ascending aorta dilatation $(\mathrm{zAscAo}<2)$. These root-only dilated patients were all men (mean age 40 years), RL-BAV and presented different degrees of aortic regurgitation and no aortic stenosis.

\section{Peak velocity and flow eccentricity}

Through-plane and magnitude velocity, jet angle and normalized displacement at proximal, mid and distal AAo are shown in Table 2. Compared to healthy control subjects, BAV patients presented higher throughplane velocity values at proximal AAo, and higher velocity magnitude, jet angle and flow displacement at the proximal and mid AAo but not in the distal part (Fig. 3). Also, RN- compared to RL-BAV presented

Table 1 Demographics and aortic dimensions in controls and bicuspid aortic valve patients

\begin{tabular}{|c|c|c|c|c|}
\hline & Healthy Controls & RL-BAV & RN-BAV & $p$-value \\
\hline N & 20 & 78 & 23 & \\
\hline Age (years) & $50.04 \pm 16.39$ & $48.43 \pm 13.15$ & $46.97 \pm 16.00$ & 0.659 \\
\hline Men (\%) & 73.30 & 62.80 & 65.20 & 0.736 \\
\hline Weight (kg) & $78.57 \pm 9.24$ & $76.17 \pm 10.38$ & $76.17 \pm 10.38$ & 0.270 \\
\hline Height (cm) & $169.42 \pm 7.73$ & $170.00 \pm 10.66$ & $172.00 \pm 9.91$ & 0.433 \\
\hline Body Surface Area $\left(\mathrm{m}^{2}\right)$ & $1.89 \pm 0.13$ & $1.83 \pm 0.21$ & $1.88 \pm 0.16$ & 0.278 \\
\hline Degree of Aortic Regurgitation (\%) & & & & 0.240 \\
\hline 0 & - & 26.8 & 35.3 & \\
\hline 1 & - & 21.1 & 5.9 & \\
\hline 2 & - & 49.3 & 47.0 & \\
\hline 3 & - & 2.8 & 11.8 & \\
\hline Degree of Aortic Stenosis (\%) & & & & 0.695 \\
\hline Absent & - & 92.9 & 90 & \\
\hline Mild & - & 5.7 & 10 & \\
\hline Moderate & - & 1.4 & 0 & \\
\hline Maximum aortic velocity (m/s) & $97.34 \pm 19.0$ & $111.15 \pm 24.35$ & $119.32 \pm 21.22$ & 0.067 \\
\hline Mean pressure gradient (mm Hg) & $3.24 \pm 1.21$ & $4.9 \pm 1.13$ & $5.69 \pm 1.71$ & 0.074 \\
\hline Systolic Arterial Pressure (mm Hg) & $136.26 \pm 19.47$ & $134.18 \pm 17.32$ & $142 \pm 17.76$ & 0.064 \\
\hline Diastolic Arterial Pressure (mm Hg) & $76.73 \pm 8.85$ & $75.72 \pm 9.06$ & $79.69 \pm 9.11$ & 0.133 \\
\hline Sinus of Valsalva Diameter (mm) & $32.77 \pm 3.44$ & $36.68 \pm 5.10$ & $34.78 \pm 2.69$ & 0.796 \\
\hline Ascending Aorta Diameter (mm) & $35.58 \pm 2.96$ & $39.86 \pm 7.51$ & $40.30 \pm 6.10$ & 0.070 \\
\hline z-score sinus of Valsalva diameter & $0.01 \pm 0.84$ & $1.41 \pm 1.29$ & $0.82 \pm 1.11$ & 0.001 \\
\hline z-score ascending aorta diameter & $0.02 \pm 0.71$ & $2.87 \pm 1.71$ & $3.03 \pm 1.4$ & $<0.001$ \\
\hline
\end{tabular}

$P$-values reported are the result of ANOVA comparison between 3 groups: controls, RL-BAV and RN-BAV in continuous variables and Chi-square test for categorical variables 


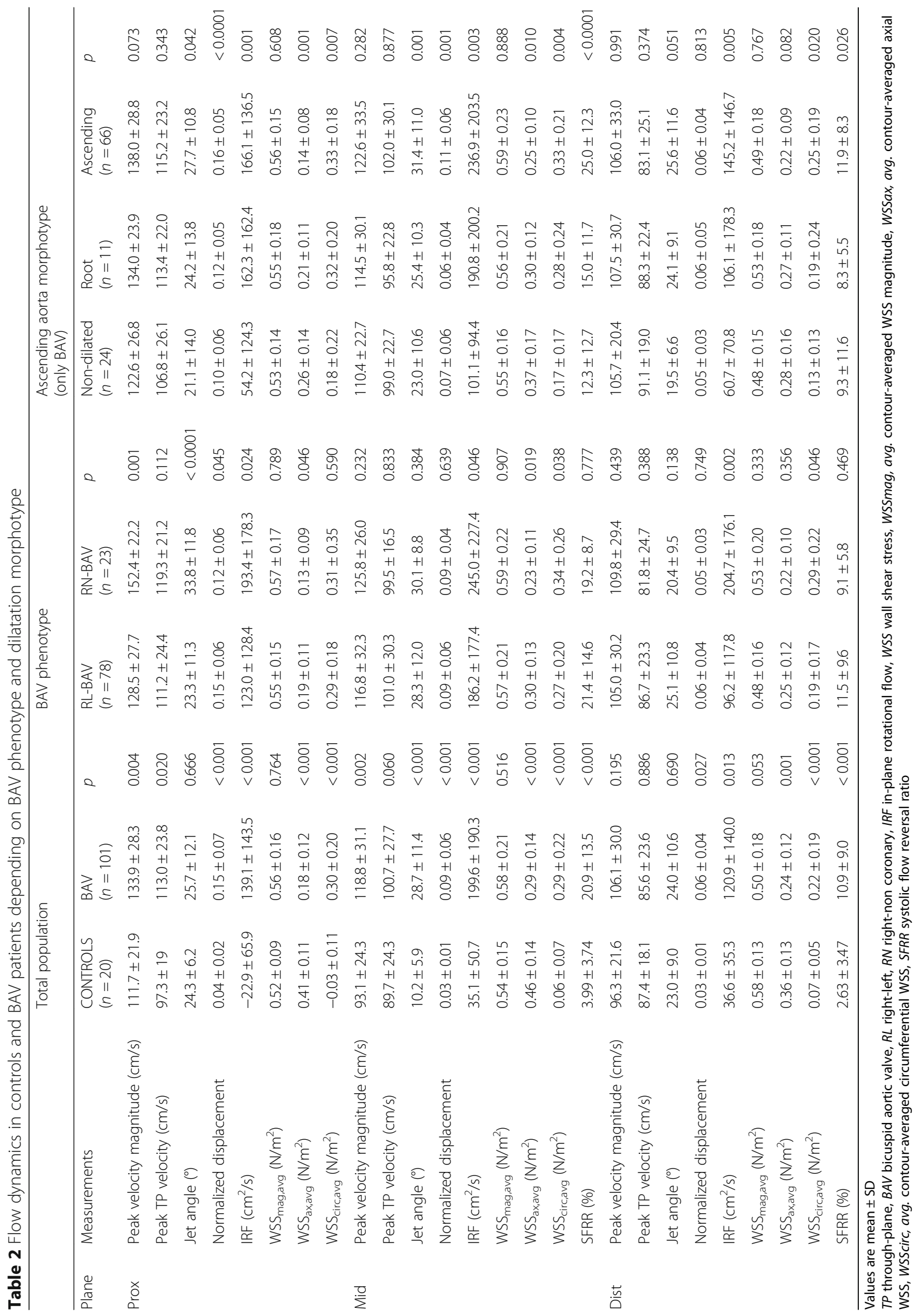




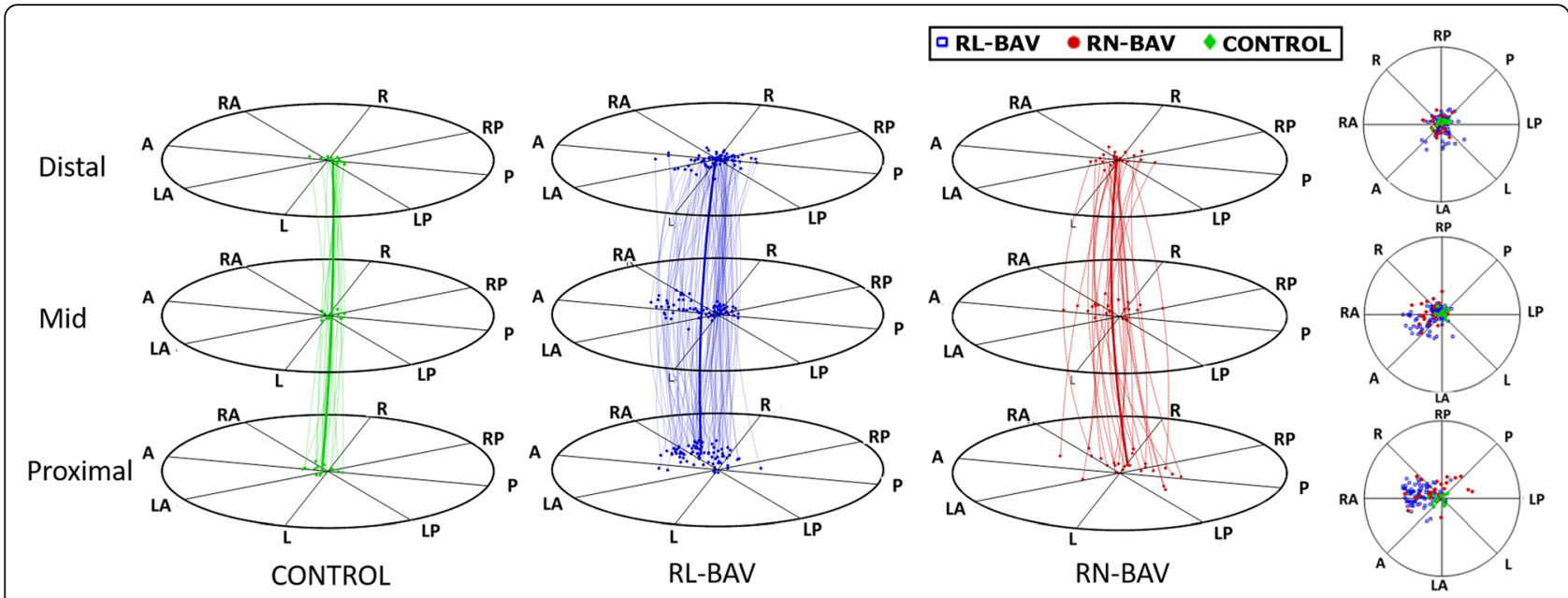

Fig. 3 Peak-systolic center of velocities. Center of velocities at proximal, mid and distal ascending aorta (AAo) for controls and BAV. Lines show the maximum velocity path along the AAo for each patient. Wider arrow represents the mean path for each group. A: anterior, L: left, P: posterior, $\mathrm{R}$ : right, $\mathrm{RL}-\mathrm{BAV}$ : right-left bicuspid aortic valve, $\mathrm{RN}-\mathrm{BAV}$ : right-non coronary bicuspid aortic valve

significant differences in peak velocity and eccentricity at proximal AAo.

When the aortic morphotype was considered in BAV, the ascending and root-morphotypes (dilated morphotype) compared to the non-dilated presented higher jet angles and displacement at the proximal and mid AAo $(P<0.05)$. However, the ascending versus the rootmorphotypes did not present differences (Table 2).

When the location of the center of velocities and flow direction were analyzed in BAV, RL-BAV presented a consistent pattern (Fig. 3), showing a right ( $28 \%$ cases) to right-anterior position (55\% cases) in the proximal aorta, with a similar profile in the mid segment and an axisymmetric profile at the distal AAo (Fig. 4a and additional movie file [Additional file 1]). However, RNBAV presented a higher variability in their flow pattern (Fig. 3) with a predominant posterior to right-posterior outflow jet ( $78 \%$ cases) in the proximal aorta that shifted to the anterior segments (55\% right-anterior and 20\% anterior) in the mid and distal AAo (Fig. 4b and additional movie file [Additional file 2]). Control patients presented non-eccentric and predominantly laminar flow (see additional movie file [Additional file 3]).

\section{In-plane rotational flow and systolic flow reversal ratio}

In-plane rotational flow (IRF) was significantly higher in RN- compared to RL-phenotype at all aortic levels. Although a trend for higher SFRR was observed in RL-BAV compared to RN-BAV, these differences did not reach statistical significance (Table 2).

Dilated BAV presented higher values of IRF and SFRR compared to healthy controls or non-dilated morphotype (Table 2, Fig. 5). IRF was mostly right-handed (98\%) in all aortic morphotypes with no statistically-significant differences. However, patients with the ascendingmorphotype presented higher IRF and SFRR than the root-morphotype at all aortic levels (Table 2) (Fig. 5).

\section{WSS and regional WSS maps}

Compared to controls, BAV presented similar magnitude $\left(\mathrm{WSS}_{\text {mag,avg }}\right)(P>0.05)$ but lower axial $\left(\mathrm{WSS}_{\mathrm{ax}, \text { avg }}\right)$

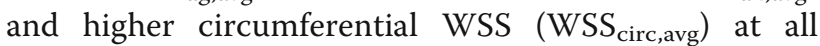
levels $(P<0.001)$ (Table 2$)$.

According to the valve phenotype, RL- compared to RN-BAV presented higher WSS $_{a x, a v g}$ in proximal and mid AAo $(P<0.05)$, whereas WSS $_{\text {circ,avg }}$ was higher in RN-phenotype in mid and distal AAo (Table 2). These differences were also observed in regional WSS maps (Figs. 6 and 7).

Based on the aortic morphotype, non-dilated BAV presented similar WSS $_{\text {mag avg }}$ and WSS $_{\text {ax,avg }}$ but higher $\mathrm{WSS}_{\text {circ,avg }}$ compared to controls at all levels $(P<0.05)$. In BAV patients, the $\mathrm{WSS}_{\text {mag,avg }}$ was similar among the different aortic morphotypes. However, $\mathrm{WSS}_{\text {ax,avg }}$ was significantly higher in the root-morphotype and $\mathrm{WSS}_{\text {circ,avg }}$ was higher in the ascending-morphotype at all levels (Table 2). Regional differences $(p<0.05)$ between morphotypes were more pronounced for circumferential than axial WSS, and were associated with regions of systolic flow reversal for axial WSS (Figs. 8 and 9).

The outflow jet direction matched the location of maximum systolic axial WSS in the aortic wall (Figs. 3 and 6). Thus, in RN-BAV, maximum systolic axial WSS extends from posterior to right-posterior proximally towards anterior to right-anterior in mid and distal AAo. However, in RL-BAV, maximum systolic axial WSS extends from right to right-anterior at all aortic levels (Figs. 6 and 7). 


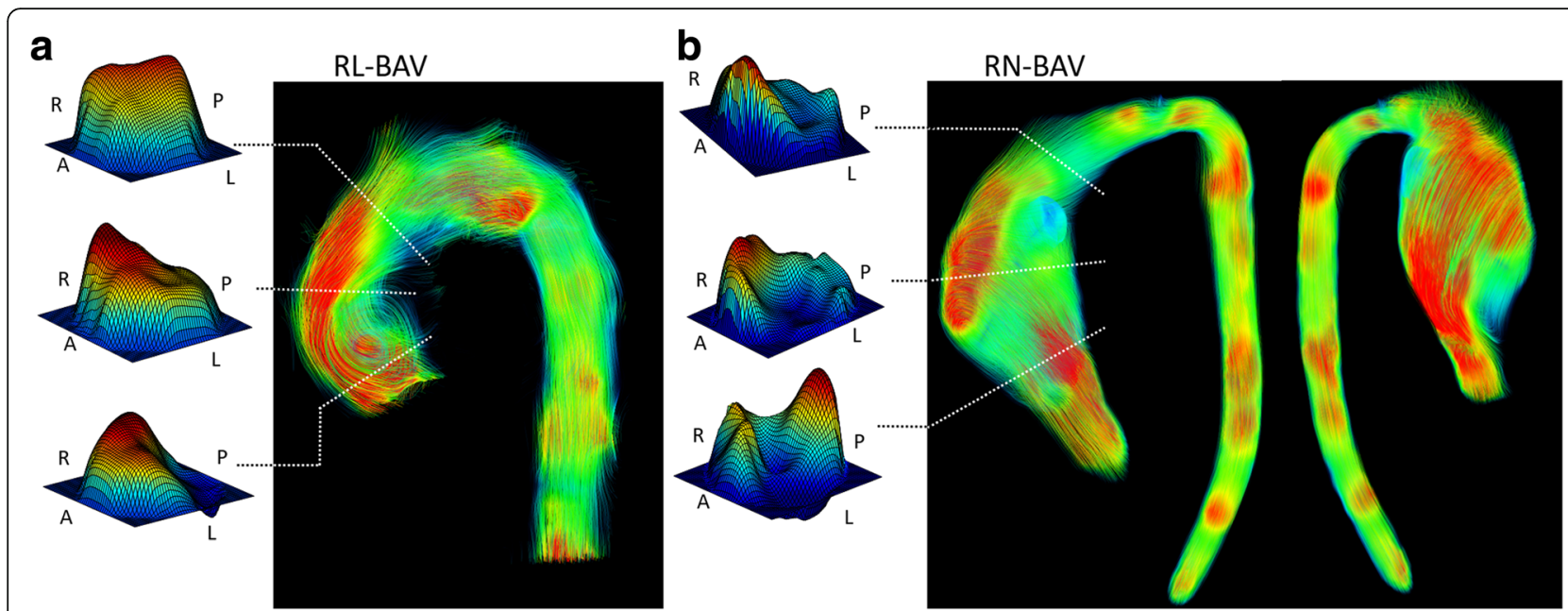

Fig. 4 Through-plane velocity profiles and streamlines in BAV. a Asymmetric outflow jet to the anterior wall in a RL-BAV. b RN-BAV with a posterior outflow jet shifting to the anterior wall at mid and distal ascending aorta. Abbreviations as in Fig. 3

\section{Correlates of root or ascending-morphotypes}

Significant bivariable (unadjusted) and multivariable adjusted correlates of aortic dilatation ( $>2$ z-score) [26] in BAV are listed in Table 3 (variables selected from the 4D-flow-derived variables listed in Table 2 and including sex, age and body surface area). Displacement, IRF and WSS were transformed to their natural logarithms.

On multivariable analysis, only sex (male), natural

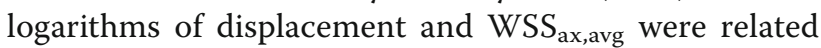
to the presence of the root-morphotype with an AUC: $0.91 \quad(P<0.001)$ (Fig. 10). However, RN-phenotype, SFRR and $\mathrm{WSS}_{\text {circ,avg }}$ in the mid AAo were related to the presence of the ascending- morphotype with an AUC: $0.89(P<0.001)$ (Table 3) (Fig. 10).

\section{Discussion}

In our study, we assessed the relation between aortic flow patterns and regional WSS components (magnitude, axial and circumferential) through the entire ascending aorta in a large BAV population. In order to avoid the effect of changes in flow dynamics and WSS secondary to aortic dilatation [29] or severe valvular disease, only BAV patients with non-severe valvular dysfunction and aortic diameters $\leq 45 \mathrm{~mm}$ were included.

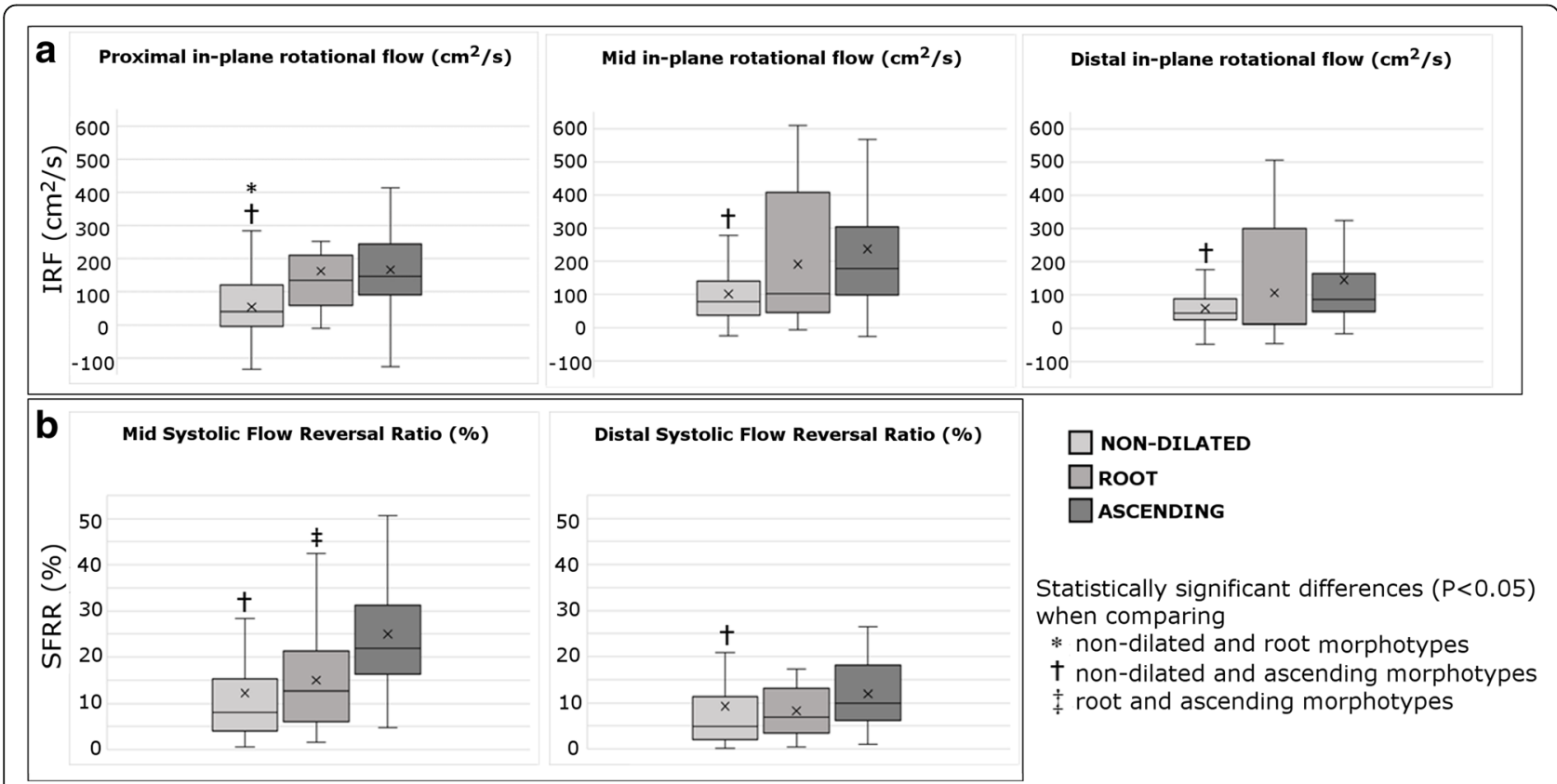

Fig. 5 In-plane rotational flow (IRF) and systolic backward flow (SFRR). a Mean IRF and b SFRR in controls and BAV, based on the dilatation morphotype 


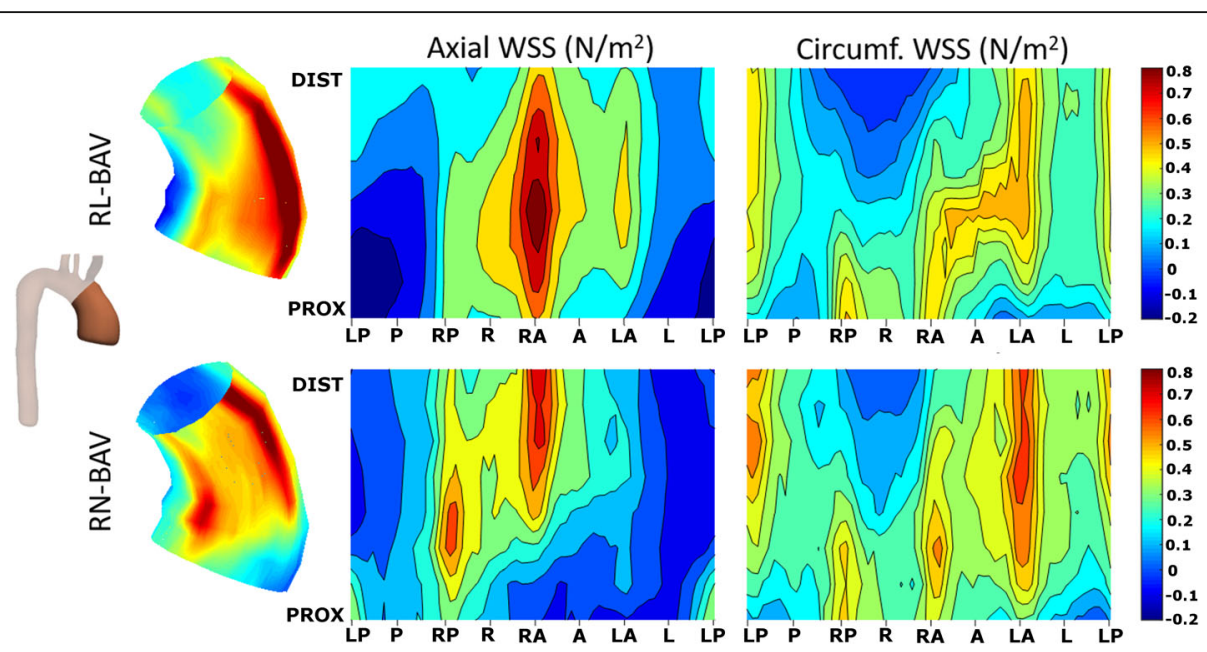

Fig. 6 Peak-systolic axial and circumferential WSS maps in RL-and RN-BAV. RL-BAV show maximum axial WSS in the right to right-anterior wall and lower values of circumferential WSS at all levels. In RN-, maximum axial WSS extends from the right-posterior wall proximally to right-anterior wall at mid and distal ascending aorta, with a higher distal circumferential WSS. Prox: proximal, Dist: distal, WSS: wall shear stress, other abbreviations as in Fig. 3

Also, the specific role of flow parameters and WSS components in the ascending aorta dilatation and morphotype was assessed by unadjusted and multivariable adjusted analysis.

The main findings of our study were that: 1) RL-BAV patients present a sustained flow towards the anterior and right-anterior aortic walls, whereas, RN-BAV present a predominantly posterior output flow that shifts towards the right and right-anterior walls in the mid and distal AAo inducing an increase in the IRF. This flow distribution reflects into regional WSS patterns. 2) Sex (male), normalized displacement and axial WSS in the proximal AAo are the main factors associated with the root-morphotype, whereas RN-phenotype, SFRR and circumferential WSS are the main factors related to the ascending-morphotype.

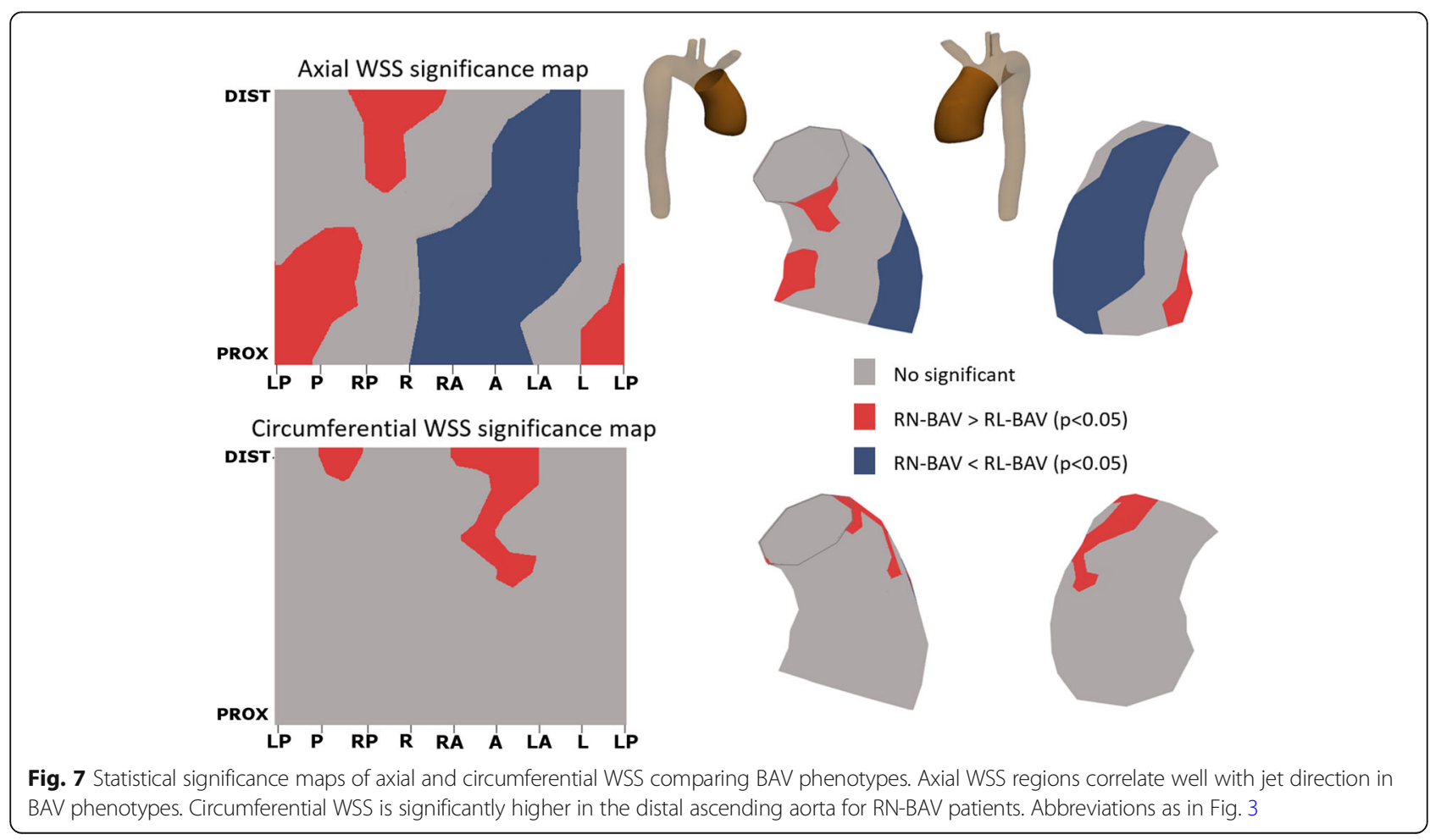




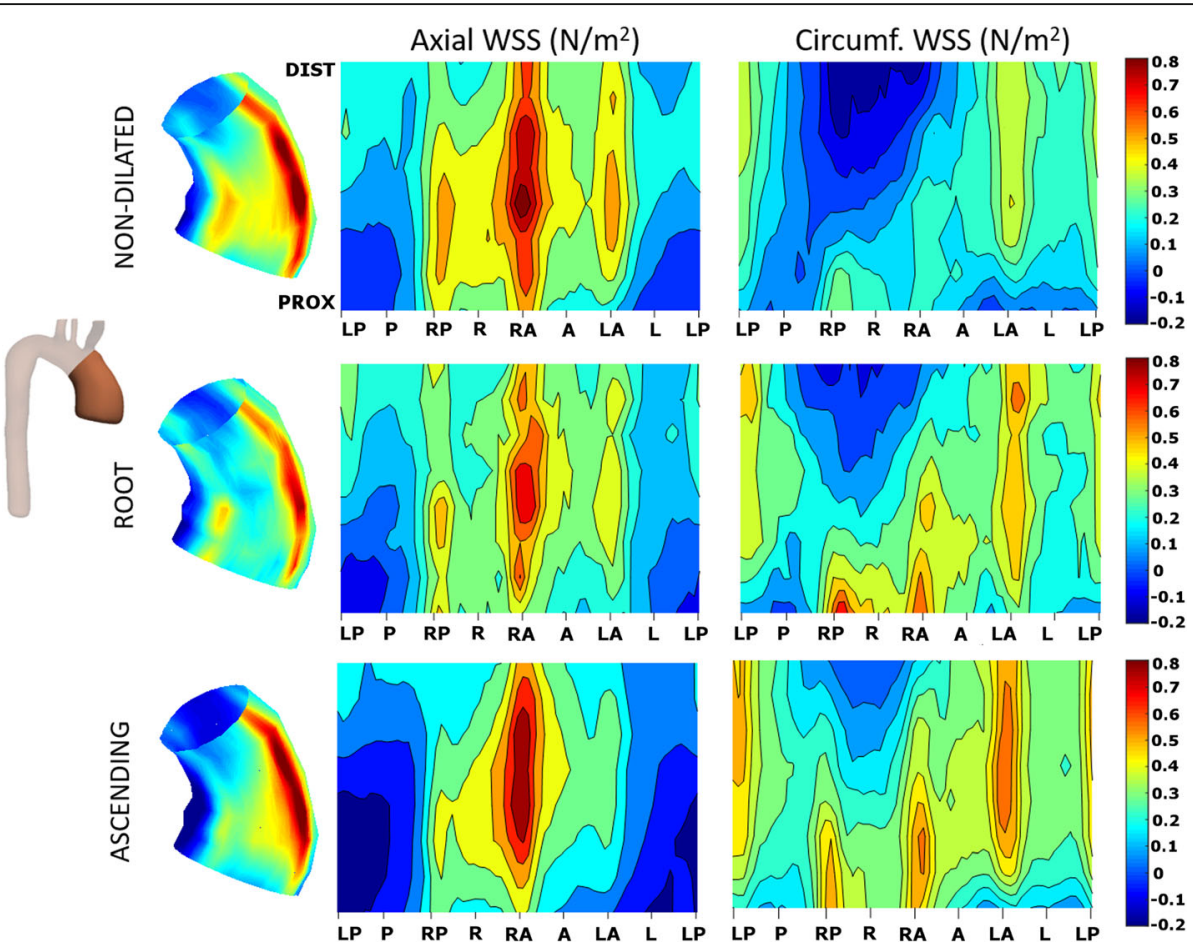

Fig. 8 Peak-systolic axial and circumferential WSS maps according to BAV aortic morphotype. Root-morphotype presents higher proximal axial WSS compared to ascending-, however, circumferential WSS is higher in ascending-morphotype at all levels. Abbreviations as in Fig. 3

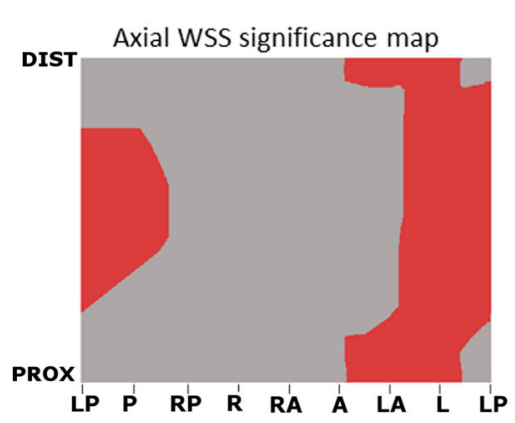

Circumferential WSS significance map

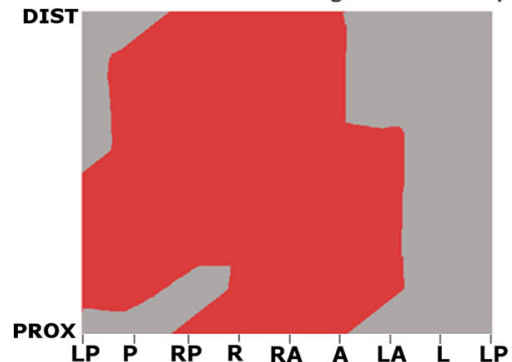

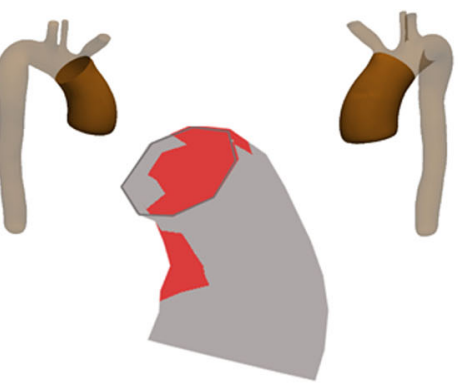

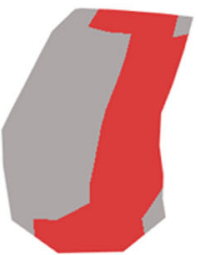

No significant

Differences among dilatation morphotypes $(\mathrm{p}<0.05)$
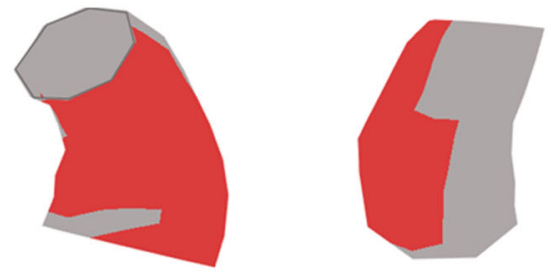

Fig. 9 Statistical significance maps of axial and circumferential WSS comparing BAV morphotypes. Statistical differences in regional WSS are more pronounced in circumferential than axial WSS, and are associated with regions of systolic flow reversal for axial WSS 
Table 3 Unadjusted and adjusted relationship of demographic and flow variables to root (top) and ascending (bottom) morphotypes

Root morphotype

Age
BSA
BAV phenotype
(RN vs RL)
Sex (male)
Proximal peak velocity magnitude $(\mathrm{cm} / \mathrm{s})$
Proximal jet angle $\left({ }^{\circ}\right)$
Ln Proximal normalized displacement
Ln Proximal IRF (cm²/s)
Ln Proximal WSS mag,avg $\left(\mathrm{N} / \mathrm{m}^{2}\right)$
Ln Proximal WSS $\mathrm{Wx}_{\text {axavg }}\left(\mathrm{N} / \mathrm{m}^{2}\right)$
Ln Proximal WSS circ,avg $\left(\mathrm{N} / \mathrm{m}^{2}\right)$
Ascending morphotype

OR

Age

BSA

BAV phenotype

(RN vs RL)

Sex (male)

Mid jet angle $\left({ }^{\circ}\right)$

Ln Mid normalized displacement

Ln Mid IRF $\left(\mathrm{cm}^{2} / \mathrm{s}\right)$

Ln Mid WSS ax,avg $\left(\mathrm{N} / \mathrm{m}^{2}\right)$

Ln Mid WSS circ,avg $\left(\mathrm{N} / \mathrm{m}^{2}\right)$

Mid SFRR (\%)

Univariate adjusted correlates of root morphotype Multivariable adjusted correlates of root morphotype $\mathrm{OR}$

$p$-value
0.530
0.104
0.005
0.050
0.719
0.490
0.040
0.002
0.761
0.081
0.243

Univariate adjusted correlates of ascending morphotype

$\mathrm{OR}$

0.99 (0.96-1.03)

$2.51(0.30-2.17)$

$1.99(1.61-3.48)$

$0.82(0.32-2.10)$

$1.09(1.03-1.14)$

$2.85(1.56-5.23)$

$2.98(1.54-5.78)$

$0.14(0.04-0.48)$

$1.51(1.16-3.68)$

$1.12(1.06-1.19)$ $p$-value

0.917

0.398

0.026

0.678

0.001

0.001

0.001

0.221

0.002

0.001
OR

$p$-value

0.291

0.998

0.011

1.25

1.1

0.009

0.79

0.940

1.130 .003

$0.02 \quad 0.673$

Multivariable adjusted correlates of ascending morphotype

OR $\quad p$-value

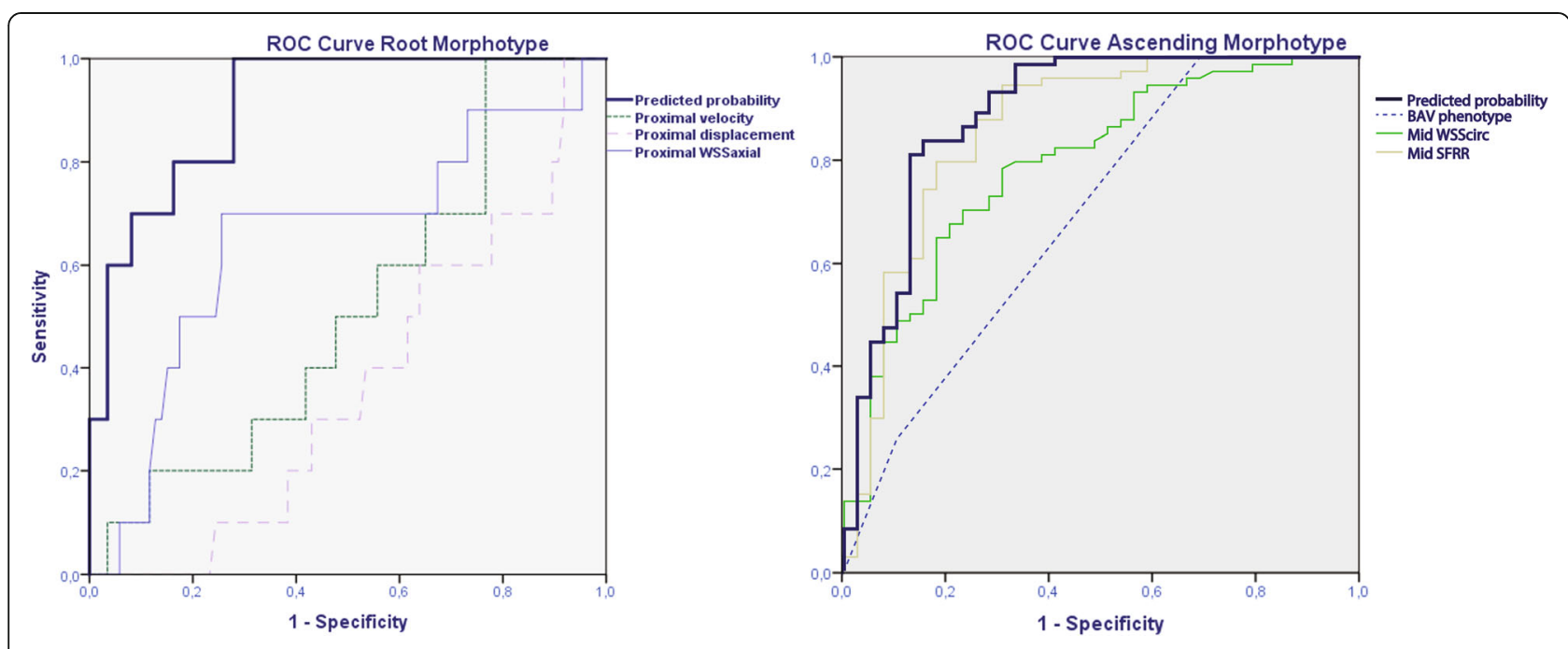

Fig. 10 ROC curves showing main factors associated with ascending aorta morphotypes. Composite probability shows the best AUC for prediction in both morphotypes. SFRR: systolic flow reversal ratio, WSScirc: circumferential WSS 
To our knowledge, this is the first large study conducted in BAV patients in which different patterns of axial and circumferential regional WSS maps were used to explain variations in the AAo dilatation morphotype. Previous studies have emphasized differences in flow variables and WSS between the RN- and RL-BAV [7, 10, $11,13,14]$ with little or no correlation with the aortic dilatation morphotype $[7,11]$. In this regard, Mahadevia et al. [7] did not analyze rotational flow or WSS components when studying BAV aortopathy, while Bissell et al. [11] only showed an increased rotational flow at larger aortic diameters suggesting a potential causative role. Thus, it is of great interest to ascertain the main factors associated with aortic dilatation since aortic diameter and structural changes in the aortic wall are related to clinical events regardless of valve phenotype $[6,8]$.

\section{Flow patterns}

Owing to the asymmetric valve opening, there is an increase in the jet angle and in the displacement of the center of velocities with respect to the center of the lumen that induces an asymmetric distribution of the WSS pattern as previously described [7, 11, 13, 14]. Similar to those of Mahadevia et al. [7], our results confirm that the jet angle is wider in RN-phenotype, whereas normalized displacement is greater in the RLphenotype. Also, we demonstrated that these variables are greater at the proximal aorta with a progressive reduction at the distal AAo that suggests that flow tends to be more symmetric in the distal AAo. Our results differ from those of Mahadevia et al. [7] since they reported flow angle and displacement to be the main factors involved in AAo dilatation. However, they determined the absolute value of the displacement, whereas we report this value normalized by aortic diameter as suggested elsewhere [22].

\section{In-plane rotational flow and SFRR}

Similar to previous studies [11, 13, 30, 31], we found that BAV presented greater rotational flow compared to controls, with the RN-phenotype being greater than in RL- at the mid and distal AAo. This finding can be justified by the fact that the flow shifts from posterior towards anterior segments in RN-BAV. This rotational flow is not only significantly greater in RN-BAV but also in those with the ascending-morphotype. An increased rotational flow induces an increase in the circumferential WSS which justifies that both parameters were statistically significant in the ascending-morphotype on univariable analysis.

In our population, most of the BAV patients presented right-handed flow (98\%). The lack of left handed helical flow seen in our study could be a sign of left handed helical flow being associated with severe/late disease process [11], and therefore, not seen in the benign aortopathy population $(\leq 45 \mathrm{~mm})$ included in our study.

The presence of retrograde flow at systole has been reported in patients with greater aortic diameters $[29,32,33]$. We found that higher values of SFRR are associated with the ascending-morphotype and not with the root-morphotype. An increase in the SFRR may induce an asymmetric increase and directional variations in the WSS contributing to dilatation. It is not clear whether this parameter is the cause or consequence of aortic dilatation; however, we observed that this craniocaudal flow also exists in BAV with normal aortic diameters. This finding suggests that this flow may act as a causal agent of aortic dilatation and would increase as the aorta dilates, thereby perpetuating this process.

\section{WSS and aortic dilatation}

Our study is consistent with previous publications [7, 13] which suggest that the magnitude of WSS lacks significance since its value is similar in controls and BAV. However, controls present increased axial WSS because of predominant laminar flow, while helical flow in BAV increases circumferential WSS [13]. Thus, the different WSS components (axial and circumferential) constitute an interesting parameter in the assessment of aortic dilatation [11].

A detailed analysis of WSS permitted us to use a 3D representation of axial and circumferential WSS maps along the AAo, showing asymmetrical patterns that may contribute to structural changes in the aortic wall (elastin and metalloproteinases) related to aortic dilatation [8]. Furthermore, the presence of eccentric but uniform flow along the anterior AAo in the RL-phenotype determines that the axial component of WSS is greater in this subgroup of patients; however, the eccentric but helical flow in the RN-phenotype determines that the circumferential component of WSS is greater in this subgroup. This variation in WSS components may also influence the aortic morphotype. Thus, patients with a greater axial component exhibit more dilatation at the aortic root; however, greater circumferential WSS is associated with dilatation in the AAo.

Despite the correlations found here and elsewhere, the causative role of the observed flow disturbances need to be assessed in longitudinal studies. In particular, it has been mainly discussed in root only dilatation in BAV, which is thought to be a predominantly genetic form of BAV disease [9]. Our data confirmed the previously found association between root (only) dilatation and male sex [2].

The association of male sex, normalized displacement, and axial WSS in the proximal aorta discriminated the root-morphotype with an AUC of 0.91. However, the combination of an RN-phenotype, circumferential WSS 
and SFRR discriminated the ascending-morphotype with an AUC of 0.89. Thus, we believe these parameters should be considered in the evaluation of BAV beyond aortic diameters. This additional information could identify patients at higher risk of aortopathy that may require a closer follow-up.

\section{Limitations}

The prospective nature of our study determined the inclusion of more patients with the RL-phenotype than $\mathrm{RN}$-; however, our cohort reflects the distribution of fusion phenotypes in the general BAV population.

Healthy subjects were recruited to match BAV patients for age and aortic diameters. Although aortic diameters were slightly larger in BAV, these differences were not statistically significant.

Owing to the limited spatial and temporal resolution of 4D-flow, WSS is known to be underestimated [25, 34, 35]. However, all acquisitions were made with the same imaging parameters and analyzed with the same methodology and previous work highlighted that regions of high/ low WSS are matched despite different spatial and temporal resolutions [35]. Additionally, manual segmentation causes intra- and inter-observer variability. Nevertheless, the robustness of WSS measurements employed in this study and their reproducibility has been previously demonstrated [36].

WSS estimation was limited to 8 slices in the AAo at peak systole. Thus, very localized regions of altered WSS may have been lost and temporal variations were not assessed. The use of a volumetric WSS method [37, 38], would allow a more detailed analysis.

Despite flow variables are likely to vary during the ejection phase, our measurements of jet angle, flow displacement, IRF and WSS were performed only at peak systole. Moreover, these measurements were performed averaging the results obtained at four successive time instants to reduce noise. Despite this approach has been used by several authors $[7,11,13]$, and have proved high reproducibility [38], it may imply the loss of possible information contained in other systolic phases.

We conducted a cross-sectional study to evaluate the impact of flow dynamics in aortic dilatation in BAV. However, the real influence of these parameters on the concurrence of aortic dilatation needs to be determined in further longitudinal studies.

\section{Conclusions}

BAV patients present altered flow patterns that vary depending on their valvular phenotype. RL-BAV patients present an anterior distribution, whereas, RN-BAV present a predominant posterior outflow jet at the sinotubular junction that shifts to anterior or right anterior in mid and distal AAo. This flow distribution induces an increase in the $\mathrm{WSS}_{\mathrm{ax}}$ avg in the anterior aortic wall in RL-BAV patients, while, an increase in the in-plane rotational flow and $\mathrm{WSS}_{\text {circ avg }}$ in the mid and distal AAo in RN-BAV patients. These results may explain different AAo dilatation morphotypes in the BAV population. Thus, in addition to aortic diameters, the assessment of the different WSS components (axial and circumferential) and derived flow parameters may contribute to identify more completely, and precisely which patients have a higher risk of aortic dilatation. The follow-up of our series will permit validation of our results.

\section{Additional files}

Additional file 1: Systolic flow in a RL-BAV patient. Systolic flow in a RLBAV patient, showing a jet toward the anterior wall of the ascending aorta. (AVI $4858 \mathrm{~kb}$ )

Additional file 2: Systolic flow in a RN-BAV patient. Systolic flow in a RNBAV patient, showing a posterior outflow jet that shifts anteriorly in the mid and distal ascending aorta. (AVI 3996 kb)

Additional file 3: Systolic flow in a healthy volunteer. Systolic flow in a healthy volunteer, showing a non-eccentric and laminar flow. (AVI 4694 kb)

\section{Abbreviations \\ 4D-flow: Time-resolved three-dimensional phase-contrast magnetic reson- ance imaging; AAo: Ascending aorta; BAV: Bicuspid aortic valve; bSSFP: Balanced steady state free precession; CMR: Cardiovascular magnetic resonance; ECG: Electrocardiogram; FOV: Field of view; IRF: In-plane rotational flow; PC: Phase contrast; RL-BAV: Bicuspid aortic valve with fusion of the right and left coronary cusps; RN-BAV: Bicuspid aortic valve with fusion of the right coronary cusp and the non-coronary cusp; ROC: Region of interest; SFRR: Systolic flow reversal ratio; VENC: Velocity encoding; WSS: Wall shear stress; WSS $_{\text {ax avg: }}$ Circumferentially-averaged axial WSS; WSS $_{\text {circ }}$ \\ avg: Circumferentially-averaged circumferential WSS; WSS mag $_{\text {in }}$ \\ avg: Circumferentially-averaged magnitude WSS}

\section{Acknowledgements}

We would like to thank Christopher François (University of Wisconsin-Madison) and Rob van der Geest (Leiden University Medical Center) for advice on 4D-flow sequences, and Roberto García Álvarez (GE Healthcare) for technical support. We are also grateful to Augusto Sao Avilés for statistical analysis and Christine O'Hara for English revisions.

\section{Funding}

This study has been funded by Instituto de Salud Carlos III through the project PI11/01081, La Marató de TV3 (project number 20151330), and by Ministerio de Economía y Competitividad through Retos-Colaboración 2016 (RTC-2016-5152-

1). Guala A. has received funding from the European Union Seventh Framework Programme FP7/People under grant agreement $n^{\circ} 267128$.

\section{Availability of data and materials}

The datasets generated and analyzed during the current study are available from the corresponding author on reasonable request.

\section{Authors' contributions}

JFR and LD: conception and design, analysis and interpretation of data, drafting the manuscript. RK, GT, LG, AG: analysis and interpretation of data. GM, FV: data collection, analysis and interpretation of data. $\mathrm{MH}$ : data collection. LGu, TG: selection of patients based on echo criteria, analysis and interpretation of data. $\mathrm{KJ}$ and OW: provided and maintained the 4D flow sequence, analysis and interpretation of data. DG: conception and design. AE: conception and design, analysis and interpretation of data. All authors critically reviewed the manuscript and approved the final version. 


\section{Ethics approval and consent to participate}

The study was approved by the local ethics committee (Hospital Universitari Vall d'Hebron) and informed consent was obtained from all participants.

\section{Competing interests}

The authors declare that they have no competing interests.

\section{Publisher's Note}

Springer Nature remains neutral with regard to jurisdictional claims in published maps and institutional affiliations.

\section{Author details}

'Hospital Universitari Vall d'Hebron, Department of Cardiology. Vall d'Hebron Institut de Recerca (VHIR), Universitat Autònoma de Barcelona, Paseo Vall d'Hebron 119-129, 08035 Barcelona, Spain. ${ }^{2}$ Cardiac Imaging Department, CETIR-ERESA, Clínica del Pilar-Sant Jordi, Barcelona, Spain. ${ }^{3}$ Departments of Medical Physics \& Radiology, University of Wisconsin - Madison, Madison, WI, USA.

\section{Received: 25 April 2017 Accepted: 9 April 2018}

Published online: 26 April 2018

\section{References}

1. Tzemos N, Therrien J, Thanassoulis G, et al. With bicuspid aortic valves. World Health. 2013:300(11):1317-25.

2. Della Corte A, Bancone C, Dialetto G, et al. The ascending aorta with bicuspid aortic valve: a phenotypic classification with potential prognostic significance. Eur J Cardio-Thoracic Surg. 2014;46(2):240-7. https://doi.org/10. 1093/ejcts/ezt621.

3. Verma S, Siu SC. Aortic dilatation in patients with bicuspid aortic valve. N Engl J Med. 2014;370(20):1920-9. https://doi.org/10.1056/NEJMra1207059.

4. Elefteriades JA, Farkas EA. Thoracic aortic aneurysm. Clinically pertinent controversies and uncertainties. J Am Coll Cardiol. 2010;55(9):841-57. https://doi.org/10.1016/j.jacc.2009.08.084.

5. Pape $L A$, Tsai $T$, Isselbacher EM, et al. Aortic diameter $>5.5 \mathrm{~cm}$ is not a good predictor of type a aortic dissection observations from the International Registry of Acute Aortic Dissection (IRAD). Circulation. 2007; 116:1120-7. https://doi.org/10.1161/CIRCULATIONAHA.107.702720.

6. Michelena HI, Khanna AD, Mahoney D, et al. Incidence of aortic complications in patients with bicuspid aortic valves. JAMA. 2011;306(10): 1104-12. https://doi.org/10.1001/jama.2011.1286306/10/1104 [pii].

7. Mahadevia R, Barker AJ, Schnell S, et al. Bicuspid aortic cusp fusion morphology alters aortic three-dimensional outflow patterns, wall shear stress, and expression of aortopathy. Circulation. 2014;129(6):673-82. https:// doi.org/10.1161/CIRCULATIONAHA.113.003026.

8. Guzzardi DG, Barker AJ, Van Ooij P, et al. Valve-related hemodynamics mediate human bicuspid aortopathy: insights from wall shear stress mapping. J Am Coll Cardiol. 2015;66(8):892-900. https://doi.org/10.1016/j. jacc.2015.06.1310.

9. Girdauskas E, Borger MA, Secknus M-A, Girdauskas G, Kuntze T. Is aortopathy in bicuspid aortic valve disease a congenital defect or a result of abnormal hemodynamics? A critical reappraisal of a one-sided argument. Eur J CardioThoracic Surg. 2011;39(6):809-14. https://doi.org/10.1016/j.jcts.2011.01.001.

10. Hope MD, Hope TA, Meadows AK, et al. Bicuspid aortic valve : fourdimensional MR evaluation of ascending aortic systolic methods : results. Radiology. 2010;255(1):53-61.

11. Bissell MM, Hess AT, Biasiolli $L$, et al. Aortic dilation in bicuspid aortic valve disease: flow pattern is a major contributor and differs with valve fusion type. Circ Cardiovasc Imaging. 2013;6(4):499-507. https://doi.org/10.1161/ CIRCIMAGING.113.000528.

12. Bieging $E T$, Frydrychowicz $A$, Wentland $A L$, et al. In vivo three-dimensional mr wall shear stress estimation in ascending aortic dilatation. J Magn Reson Imaging. 2011;33(3):589-97. https://doi.org/10.1002/jmri.22485.

13. Meierhofer C, Schneider EP, Lyko C, et al. Wall shear stress and flow patterns in the ascending aorta in patients with bicuspid aortic valves differ significantly from tricuspid aortic valves: a prospective study. Eur Heart J Cardiovasc Imaging. 2013;14:797-804. https://doi.org/10.1093/ehjci/jes273.

14. Barker AJ, Markl M, Bürk J, et al. Bicuspid aortic valve is associated with altered wall shear stress in the ascending aorta. Circ Cardiovasc Imaging 2012;5(4):457-66. https://doi.org/10.1161/CIRCIMAGING.112.973370.
15. Malek AM, Alper SL, Izumo S. Hemodynamic shear stress and its role in atherosclerosis. JAMA. 1999;282(21):2035-42. https://doi.org/10.1001/jama. 282.21.2035

16. Ruddy JM, Jones JA, Stroud RE, Mukherjee R, Spinale FG, Ikonomidis JS. Differential effect of wall tension on matrix metalloproteinase promoter activation in the thoracic aorta. J Surg Res. 2010;160(2):333-9. https://doi. org/10.1016/j.jss.2008.12.03350022-4804(08)01590-4 [pii].

17. van Ooij P, Potters WW, Collins J, et al. Characterization of abnormal wall shear stress using 4D flow MRI in human bicuspid aortopathy. Ann Biomed Eng. 2015;43(6):1385-97. https://doi.org/10.1007/s10439-014-1092-7.

18. Gu T, Korosec FR, Block WF, et al. PC VIPR: a high-speed 3D phase-contrast method for flow quantification and high-resolution angiography. AJNR Am J Neuroradiol. 2005;26(4):743-9.

19. Johnson KM, Markl M. Improved SNR in phase contrast velocimetry with five-point balanced flow encoding. Magn Reson Med. 2010;63:349-55. https://doi.org/10.1002/mrm.22202.

20. Johnson KM, Lum DP, Turski PA, Block WF, Mistretta CA, Wieben O. Improved 3D phase contrast MRI with off-resonance corrected dual Echo VIPR. Magn Reson Med. 2009;60(6):1329-36. https://doi.org/10.1002/mrm. 21763.Improved.

21. Markl M, Frydrychowicz A, Kozerke S, Hope MD, Wieben O. 4D flow MRI. J Magn Reson Imaging. 2012;36(5):1015-36. https://doi.org/10.1002/ jmri.23632.

22. Sigovan M, Hope MD, Dyverfeldt P, Saloner D. Comparison of fourdimensional flow parameters for quantification of flow eccentricity in the ascending aorta. J Magn Reson Imaging. 2011;34(5):1226-30. https://doi.org/ 10.1002/jmri.22800.

23. Hess AT, Bissell MM, Glaze SJ, et al. Evaluation of circulation, $\Gamma$, as a quantifying metric in 4D flow MRI. J Cardiovasc Magn Reson. 2013;15(Suppl 1):E36. https://doi.org/10.1186/1532-429X-15-S1-E36.

24. Bensalah MZ, Bollache E, Kachenoura N, et al. Geometry is a major determinant of flow reversal in proximal aorta. Am J Physiol Heart Circ Physiol. 2014;306:1408-16. https://doi.org/10.1152/ajpheart.00647.2013.

25. Stalder AF, Russe MF, Frydrychowicz A, Bock J, Hennig J, Markl M. Quantitative 2D and 3D phase contrast MRl: optimized analysis of blood flow and vessel wall parameters. Magn Reson Med. 2008;60(5):1218-31. https://doi.org/10.1002/mrm.21778.

26. Campens L, Demulier L, De Groote K, et al. Reference values for echocardiographic assessment of the diameter of the aortic root and ascending aorta spanning all age categories. Am J Cardiol. 2014;114(6):91420. https://doi.org/10.1016/j.amjcard.2014.06.024.

27. Whittaker J, Somers M, Whitehead C. The netlog transformation and quantile regression for the analysis of a large credit scoring database. Journal R Stat Soc Ser C. 2005;54(5):863-78. https://doi.org/10.1111/j.14679876.2005.00520.x.

28. Evangelista A, Gallego P, Calvo-Iglesias F, et al. Anatomical and clinical predictors of valve dysfunction and aortic dilation in bicuspid aortic valve disease. Heart. 2017; https://doi.org/10.1136/heartjnl-2017-311560.

29. Bürk J, Blanke P, Stankovic Z, et al. Evaluation of 3D blood flow patterns and wall shear stress in the normal and dilated thoracic aorta using flowsensitive 4D CMR. J Cardiovasc Magn Reson. 2012;14(1):84. https://doi.org/ 10.1186/1532-429X-14-84.

30. Lorenz R, Bock J, Barker AJ, et al. 4D flow magnetic resonance imaging in bicuspid aortic valve disease demonstrates altered distribution of aortic blood flow helicity. Magn Reson Med. 2014;71(4):1542-53. https://doi.org/ $10.1002 / \mathrm{mrm} .24802$.

31. Hope MD, Hope TA, Crook SES, et al. 4D flow CMR in assessment of valverelated ascending aortic disease. JACC Cardiovasc Imaging. 2011;4(7):781-7. https://doi.org/10.1016/j.jcmg.2011.05.004.

32. Barker AJ, Lanning C, Shandas R. Quantification of hemodynamic wall shear stress in patients with bicuspid aortic valve using phase-contrast MRI. Ann Biomed Eng. 2010;38(3):788-800. https://doi.org/10.1007/s10439-009-9854-3.

33. Hope TA, Markl M, Wigström L, Alley MT, Miller DC, Herfkens RJ. Comparison of flow patterns in ascending aortic aneurysms and volunteers using fourdimensional magnetic resonance velocity mapping. J Magn Reson Imaging. 2007;26(6):1471-9. https://doi.org/10.1002/jmri.21082.

34. Dyverfeldt P, Bissell MM, Barker AJ, et al. 4D flow cardiovascular magnetic resonance consensus statement. J Cardiovasc Magn Reson. 2015;17(1):72. https://doi.org/10.1186/s12968-015-0174-5.

35. Cibis M, Potters W, Gijsen FJ, et al. The effect of spatial and temporal resolution of cine phase contrast MRI on wall shear stress and oscillatory 
shear index assessment. PLoS One. 2016;11(9):1-15. https://doi.org/10.1371/ journal.pone.0163316.

36. Markl M, Wallis W, Harloff A. Reproducibility of flow and wall shear stress analysis using flow-sensitive four-dimensional MRI. J Magn Reson Imaging. 2011;33(4):988-94. https://doi.org/10.1002/jmri.22519.

37. Sotelo J, Urbina J, Valverde I, et al. 3D quantification of wall shear stress and oscillatory shear index using a finite-element method in 3D CINE PC-MRI data of the thoracic aorta. IEEE Trans Med Imaging. 2016;48(10):1-1. https:// doi.org/10.1109/TMI.2016.2517406.

38. Van Ooij P, Powell AL, Potters WV, Carr JC, Markl M, Barker AJ.

Reproducibility and interobserver variability of systolic blood flow velocity and $3 \mathrm{D}$ wall shear stress derived from $4 \mathrm{D}$ flow MRI in the healthy aorta. J Magn Reson Imaging. 2016;43(1):236-48. https://doi.org/10.1002/jmri.24959.

Ready to submit your research? Choose BMC and benefit from:

- fast, convenient online submission

- thorough peer review by experienced researchers in your field

- rapid publication on acceptance

- support for research data, including large and complex data types

- gold Open Access which fosters wider collaboration and increased citations

- maximum visibility for your research: over $100 \mathrm{M}$ website views per year

At BMC, research is always in progress.

Learn more biomedcentral.com/submissions 